\title{
El Sistema Regional de Salud de San Martín y su política de reducción de la desnutrición infantil: aplicación en el Perú del Enfoque de Evaluación de Sistemas de Salud/Health Systems Assessment
}

San Martin Regional Health System policy in reduction of child malnutrition: application in Peru of the Health Systems Assessment approach

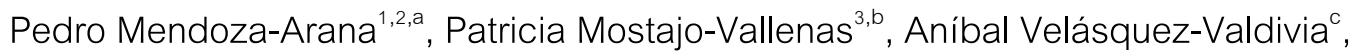 \\ Armando Cotrina-Rabanal ${ }^{4, d}$, Miguel Jaramillo-Bahanante ${ }^{5, e}$ \\ 1 Universidad Nacional Mayor de San Marcos, Lima, Perú. \\ ${ }^{2}$ Academia Nacional de Medicina, Perú. \\ ${ }^{3}$ Proyecto Evaluations de USAID Peru. \\ ${ }^{4}$ USAID Perú. \\ ${ }^{5}$ Grupo de Análisis para el Desarrollo - GRADE. \\ ${ }^{a}$ Doctor en Medicina; ${ }^{b}$ Licenciada en Economía; ${ }^{\circ}$ Médico Epidemiólogo; ${ }^{d}$ Magister en Salud Pública; ${ }^{\text {e }}$ Doctor en Economía.
}

\begin{abstract}
Resumen
Introducción. La desnutrición infantil es uno de los principales problemas de salud pública nacional y global. Objetivos. Analizar los logros y limitaciones en una Región del Perú de una política regional en la lucha contra la desnutrición. Diseño. Estudio cualitativo empleando el Enfoque de Evaluación de Sistemas de Salud - Health System Assessment. Lugar. La Región San Martín, Perú. Participantes. Gestores y funcionarios de la Región de Salud, usuarios y no usuarios de los servicios. Intervenciones. Entre junio y julio 2014 se realizaron entrevistas semiestructuradas a gestores y funcionarios de los niveles regional y local de la autoridad de salud de la Región San Martín, Perú, así como entrevistas a usuarios y no usuarios de los servicios. Principales medidas de resultados. Nudos críticos en la reducción de la desnutrición infantil. Resultados. Se identificó nudos críticos a nivel de cada uno de los seis componentes del modelo empleado: gobernanza, financiamiento, provisión de servicios, recursos humanos, medicamentos, y sistema de información. Conclusiones. Se extraen lecciones aprendidas sobre la base de los resultados, validadas en talleres con los propios funcionarios de la región y directivos nacionales de salud.
\end{abstract}

Palabras clave. Sistemas de Salud; Análisis de los Sistemas de Salud; Investigación en Sistemas de Salud.

Abstract

Introduction: Chronic infant malnutrition is one of the main public health issues in Peru and in the world. Objectives: To determine achievements and limitations of a regional policy against chronic infant malnutrition in a Region of Peru. Design: Qualitative study, using the Health System Assessment Approach, as promoted by the World Health Organization. Setting: San Martin Region, Peru. Participants: Managers and professionals working at the Health Region as well as users and non users of the services. Interventions: In June and July 2014 semistructured interviews were conducted with health managers and workers at regional and local levels as well as with services users and non users. Main outcome measures: Bottlenecks in the reduction of child malnutrition. Results: Bottlenecks for each of the model components -governance, financing, provision, health resources, drugs, and information system- were identified. Conclusions: Learned lessons from the results and validated in workshops with the participants are drawn.

Keywords: Health Care System; Health System Assessment; Health System Research.

An Fac med. 2015;76(3):269-76 / http://dx.doi.org/10.15381/anales.v76i3.11238 


\section{INTRODUCCIÓN}

La desnutrición crónica infantil (DCI) se ha convertido en un indicador clave de desarrollo de los países y ha ganado consenso a nivel global, como se aprecia tanto por su presencia entre los Objetivos de Desarrollo del Milenio ${ }^{(1)}$ como por su ubicación actual en los preliminares de la Agenda de Desarrollo Sostenible Post-2015 (2). En el Perú, es parte sustantiva de todos los documentos de consenso político, desde el Acuerdo Nacional, espacio en el cual todos los partidos políticos dialogan en torno al desarrollo e implementación de políticas de estado ${ }^{(3)}$, hasta recientes Planes que vienen siendo generados desde diferentes sectores del Estado.

Es indudable que este consenso tiene que ver con la preocupación por el desarrollo humano desde el punto de vista consensual y de derechos básicos como justicia e inclusión social, así como con los aspectos vinculados a la competitividad nacional y a las perspectivas de desarrollo asociadas a un mejor desarrollo intelectual de las nuevas generaciones ${ }^{(4)}$, las que son requeridas para una mayor competitividad nacional y subnacional ${ }^{(5)}$.

Actualmente se cuenta con intervenciones de efectividad demostrada para reducir la desnutrición crónica infantil, que han sido puestas en práctica de manera exitosa a nivel internacional ${ }^{(6)}$ y en nuestro país ${ }^{(7)}$.

Sin embargo, es claro que una lucha exitosa contra la DCI requiere no solamente de intervenciones efectivas desde el punto de vista sanitario, sino también de conocimiento acerca de cómo introducirlas y sostenerlas en los sistemas de salud ${ }^{(8)}$. A su vez, el conocimiento necesario para este proceso tiene que ver con una comprensión sistémica de los sistemas de salud, así como de una correcta identificación de los cuellos de botella que pueden ir surgiendo al implementar las políticas, y de cómo resolverlos, convertidos en lecciones aprendidas para los equipos de decisiones y de ejecutores de políticas ${ }^{(9)}$.
Hay diversas formas de identificar nudos críticos en procesos o sistemas, tales como el propuesto por Tanahashi, en $1978^{(10)}$, que con adecuaciones y actualizaciones sigue siendo empleado hasta la actualidad ${ }^{(11)}$. Sin embargo, en años recientes se ha empleado también de manera importante para analizar los sistemas de salud, el modelo propuesto por la OMS para la Evaluación de los Sistemas de Salud ${ }^{(12)}$.

El Perú es un país con un sistema de salud que se encuentra en proceso de descentralización desde el año 2002, cuando se modificó la Constitución en dicho sentido ${ }^{(13)}$, y como parte de este se han constituido autoridades de salud subnacionales, denominadas Direcciones Regionales de Salud o DIRESA, con autonomía para diseñar sus políticas subnacionales de salud bajo los lineamientos de la autoridad nacional, el Ministerio de Salud. Se estableció como política nacional la transferencia de las competencias y funciones de los diversos sectores del Estado, entre ellos, las funciones del sector salud, la misma que se inició en 2005 y concluyó en 2008 mediante decretos supremos para la transferencia a gobiernos subnacionales y municipalidades ${ }^{(14)}$, y la transferencia de la atención primaria de salud ${ }^{(15)}$ y la educación básica ${ }^{(16)}$ a estas últimas.

Existen reportes que muestran que la descentralización desarrollada en los países afecta la efectividad y el desempeño de los programas de salud pública, particularmente cuando los programas verticales se vuelven más horizontales ${ }^{(17-20)}$.

Una evaluación de las mayores iniciativas de salud ${ }^{(21)}$ indica que la introducción de gran cantidad de recursos orientados a enfermedades específicas en sistemas de salud con limitados recursos humanos, inadecuado financiamiento y gerencia del sistema, o con deficientes sistemas de información o logística, podría no garantizar los resultados esperados.

Esta situación se explica en parte por las limitaciones y deficiencias de los sis- temas de salud y porque se ha prestado poca atención en implementar estos programas e intervenciones efectivas según la disponibilidad real de recursos económicos, técnicos y de infraestructura en países de escasos recursos ${ }^{(22)}$. Para expandir las intervenciones en las áreas más pobres es necesario conocer cuáles son las restricciones ${ }^{(23)}$, su nivel de importancia, así como el grado en que la restricción puede ser tratada o reducida.

San Martín es una de las 25 regiones en que está organizado el Perú. Se encuentra en la zona oriental del país, con una extensión de $51253,87 \mathrm{~km}^{2}$ y una población total de 806452 habitantes. Su clima corresponde al tropical de la zona amazónica. Por su geografía tiene zonas de difícil acceso a la atención de salud, con población muy dispersa, siendo su densidad poblacional promedio de 3,14 habitantes $/ \mathrm{km}^{2}$. Desde el punto de vista económico, un 30\% de su población se ubica debajo de la línea de pobreza, con un PBI per cápita de 1177 dólares ${ }^{(24)}$.

El Gobierno Sub-Nacional de San Martín ha destacado entre otros gobiernos sub-nacionales en Perú, pues a pesar de estas condiciones adversas y de la escasez de recursos ha mostrado logros claros en lo relativo a la lucha contra la DCI. En el periodo 2009-2013, la DCI ha sido reducida en 11,3 puntos porcentuales (de $26,8 \%$ a $15,5 \%$ ), según la Encuesta Nacional de Demografía y Salud - ENDES 2009-2010 y 2012 . $2013^{(25,26)}$, mientras que a nivel nacional la reducción en ese mismo periodo fue de 7,6 puntos (de $25,1 \%$ a $17,5 \%$ ).

El aspecto más característico del enfrentamiento de la DCI en San Martín fue su iniciativa de conformar el Programa de Acciones Integrales para Mejorar la Nutrición Infantil - PAIMNI.

En este sentido, interesa un acercamiento de mayor detalle a cómo operó este proceso, así como a los cuellos de botella que lograron ser superados en la lucha contra la DCI, y las lecciones aprendidas de ello, las que pueden ser un insumo importante para el diseño 
e implementación de políticas de nivel subnacional, nacional, y supranacional.

\section{MÉTODOS}

Se llevó a cabo una Evaluación del Sistema de Salud de la Región San Martín empleando como esquema ordenador el Enfoque de Evaluación de Sistemas de Salud / Health System Assessment Approach ${ }^{(27)}$, que propone que un sistema de salud puede ser examinado analizando seis bloques o componentes:

- Liderazgo y Gobernanza

- Financiamiento de los Servicios de Salud

- Provisión de Servicios

- Recursos Humanos para la Salud

- Productos Médicos, Vacunas y Tecnología

- Sistemas de Información para la Salud.

Esta evaluación consideró la reducción de la desnutrición crónica infantil (DCI) como el resultado principal de interés. Para el análisis de los diferentes componentes de sistema de salud se seleccionó la entrega de cinco de las 14 intervenciones efectivas para la reducción de la desnutrición crónica infantil: control prenatal, inmunizaciones, control de crecimiento y desarrollo del niño, manejo adecuado de la enfermedad diarreica aguda y promoción de la salud (incluyendo lactancia materna exclusiva, lavado de manos, suplementación con multimicronutrientes). El análisis de los componentes del sistema sub-nacional de salud recogió información de los siguientes niveles del sistema:

- Gobierno Subnacional - Dirección Regional de Salud - DIRESA.

- Un hospital local.

- Tres Redes de Salud (Moyobamba, Bellavista y Tocache).

- Cinco Microredes de Salud - 16 establecimientos de Salud.
- 8 Municipalidades (Gobiernos locales).

- Población (usuarios y no usuarios: Se realizaron un total de 183 entrevistas).

Los instrumentos de evaluación fueron diseñados para cada uno de los seis componentes o bloques antes mencionados. Se usaron cuestionarios estructurados para entrevistar a informantes clave en uno de los niveles descritos. En el nivel Microred y Establecimientos de Salud, se utilizaron listas de verificación para la observación estructura$\mathrm{da}$, con el fin de identificar atributos en algunos de los bloques del Sistema de Salud, como los sistemas de información e inventarios de medicinas.

En lo referido a las lecciones aprendidas, se emplea la definición adoptada por las Naciones Unidas: "Una lección aprendida es conocimiento o comprensión ganadas por experiencia" (28). La experiencia puede ser positiva, como una misión exitosa, o negativa. La lección debe ser significativa en que tuvo un impacto real sobre las operaciones; válida, en que es factual o técnicamente correcta; y aplicable, en que identifica un proceso, decisión o diseño al cual puede ser aplicada”. Destacan dos aspectos: a) Uno, que la lección aprendida deviene de la experiencia, no del análisis o confrontación de lo observado con una determinada teoría; b) Dos, que debe identificar a qué decisión es aplicable, es decir, teniendo una formulación genérica, no debe ser tan general que no identifique su aplicación ${ }^{(29)}$.

Tanto los cuellos de botella como las lecciones aprendidas identificadas por el equipo investigador fueron validados en dos talleres, uno de ellos con personal y funcionarios de San Martín, y uno desarrollado con técnicos y funcionarios del Nivel Nacional.

\section{RESULTADOS}

\section{Liderazgo y Gobernanza}

San Martín viene desarrollando una propuesta de cambios en el sistema de salud, uno de cuyos elementos centrales es la separación entre la gestión administrativa y la gestión sanitaria. Esto se expresa en la decisión de que la gestión administrativa esté a cargo de cinco gerentes en las llamadas Oficinas de Operaciones, sirviendo a los Gerentes de Red. Esto permitiría recurrir a perfiles de mayor especialización y ofrecer, como lo ha hecho San Martín, mejores condiciones salariales, recurriendo para ello al cuerpo de Gerentes Públicos conducido por SERVIR. Si bien el tiempo es aún corto para evaluar el efecto de esta decisión, su lógica es consistente con la noción de separación y especialización de roles.

Por otro lado, la DIRES muestra limitaciones para involucrar actores sociales autónomos, tales como las universidades y los colegios profesionales. La articulación con universidades y colegios es importante, pues la experiencia internacional muestra que una mejor formación de pregrado así como la certificación periódica -que significan oportunidades de formación y capacitación- pueden ser parte de estrategias efectivas de retención de recursos humanos en zonas de baja densidad de recursos humanos. Al examinar este punto con los actores regionales, se consideró que un espacio de oportunidad lo constituye el Consejo Regional de Salud, del cual forman parte las universidades, y que se podría dinamizar. En la experiencia de espacios similares como el Sistema Nacional de Pregrado en Salud (SINAPRES), el aprovechamiento de estos espacios para fines estratégicos requiere un proceso de normalización de los procesos operativos ${ }^{(30)}$, por lo que la propuesta se constituye en una opción de mediano plazo.

\section{Financiamiento de la Salud}

En términos globales, el Gobierno Regional San Martín logró en el año evaluado un mayor monto de recursos: el PIM del año 2013 fue 46\% superior al PIM del 2012.

Los nudos críticos tienen que ver con el proceso de asignación de recur- 
sos, ya que los montos de presupuesto se definen finalmente en el nivel central (MEF) en función de la asignación histórica y no en función de los resultados sanitarios que se quieren obtener. En este sentido, el presupuesto asignado es inferior al solicitado. Así, las asignaciones presupuestales de las Oficinas de Operaciones/Unidades Ejecutoras - UE a las Redes, y desde allí a los niveles inferiores del sistema de salud, deben ajustarse tras recibirse el presupuesto aprobado. Igualmente, las UEs reproducen de cierta manera el comportamiento que tiene el MEF para con ellas, y desembolsan recursos a las unidades prestadoras a partir de criterios discrecionales, antes que en función a metas. En este contexto y sin mayor guía para la priorización, el nivel local no logra modificar de manera consonante sus metas y actividades al presupuesto realmente aprobado. Esto explica la necesidad identificada por nuestras entrevistas a nivel de Redes y de Microrredes de contar con personal e insumos adicionales a los propios del sector, para lograr abastecer a una demanda existente y alcanzar las metas ya comprometidas en el Plan Operativo. El personal de enfermería para la atención de CRED y el personal médico para la atención de EDA no pueden cubrirse con el presupuesto sectorial, siendo la salida de contingencia la coordinación con el Gobierno Local para que este asuma dichas obligaciones, financiando ya sea la contratación de personal o bonificaciones. En la actualidad, se cuenta con enfermeras contratadas por varias municipalidades distritales, las mismas que se incorporan al establecimiento, son capacitadas por la Red y Microred, y pierden su vinculación con el gobierno local, de manera tal que este, a pesar de financiar sus plazas, carece de mecanismos de supervisión de sus actividades.

Además, las unidades prestadoras, manifiestan tener necesidades de material logístico como vehículos, combustible, computadoras, tinta para las impresoras, entre otros, necesarios para poder cumplir con los requerimientos en sistematización de la información, la prestación del servicio en zonas alejadas o la realización de actividades de educación sanitaria.

Por otro lado, en el mediano plazo, la expectativa de los niveles locales por un proceso de formulación presupuestal aparentemente más participativo puede generar desaliento y ser contraproducente, al verificarse que, por un lado, su participación aún es muy limitada, y por otro lado, la financiación continúa determinada básicamente por la asignación histórica. Esta experiencia negativa ya se dio en los procesos de formulación de planes locales de salud de los CLAS ${ }^{(30)}$.

\section{Recursos Humanos en Salud}

En términos relativos, la brecha del personal de enfermería y de medicina está en un $60 \%$ de la dotación requerida. Sin embargo, en nuestro trabajo de campo, todas las usuarias entrevistadas que habían acudido al control de CRED comunicaron haber sido atendidas por una enfermera, mientras que solo el $50 \%$ de las madres que buscaron atención médica para su niño con EDA fueron atendidas por un médico, por lo que se puede afirmar que en términos funcionales, la brecha de médicos es más grave que la de enfermeros. El principal nudo crítico detectado es la alta rotación de personal, lo que torna inefectiva cualquier política de reducción de la brecha de recursos humanos en base a nuevas contrataciones y limita seriamente el impacto de la capacitación.

Frente a esta brecha, el plan de la región se enfoca en nuevas contrataciones e incentivos pecuniarios y no pecuniarios. El plan de reducción de la brecha de recursos humanos es ambicioso, pues prevé reducir la brecha de médicos y enfermeras en más de $80 \%$ y la de obstetras en $100 \%$. Pero sin duda encontrará problemas, ya que no ataca las condiciones que generan la alta rotación, a saber, condiciones inadecuadas en zonas alejadas y falta de incentivos para el desarrollo profesional.
Una oportunidad importante la constituye el Plan de Desarrollo de las Personas (PDP) del primer nivel de atención, el mismo que fue aprobado en febrero 2013, y constituyó una base para formular el PDP 2014. El PDP incluye actividades concretas de capacitación vinculada a competencias del personal en las intervenciones diseñadas para reducir la DCI, tales como la consejería nutricional por ciclo de vida, el control de crecimiento y desarrollo integral del niño, y la gestión de programas de orientación al personal nuevo.

Otra oportunidad es la presencia de la Universidad de la Región. La formación del personal en servicio vinculada a una certificación progresiva conducente a la especialización es una medida que podría ser coordinada con la Universidad, como por ejemplo mediante la implementación del Programa de Formación en Salud Familiar (PROFAM), que implica la formación del equipo básico a nivel de Diplomaturas, y es convalidable para una especialización; ello contribuiría a reducir la deserción del personal, que es una de las deficiencias críticas, en especial en las zonas rurales y más en el caso de los médicos.

\section{Sistemas de Información en Salud}

Actualmente el Sistema de Información de San Martín se caracteriza por la separación de sus dos plataformas básicas, la del sistema de información del SIS y la del HIS. Incluso institucionalmente se consagra esta separación al estar el primero gestionado por la Oficina de Seguros en Salud, mientras que el segundo está en manos de la Oficina de Gestión de la Información. Además, debido a la puesta en marcha de diversos proyectos e iniciativas, San Martín tiene una amplia gama de aplicativos para fines específicos, en diferentes ámbitos de la salud y para distintas áreas geográficas de la región. Un nudo crítico importante es el referido a la necesidad de recursos humanos especializados (programadores, supervisores de calidad y personal para ingreso de datos), tanto a nivel regional como local. Otro es la 
falta de disponibilidad de hardware, que se atribuye al bajo presupuesto para realizar actividades no relacionadas directamente con resultados sanitarios.

Un tercer obstáculo es la falta de circulación de información a nivel local, tanto desde los establecimientos o microrredes hacia los gobiernos locales, como desde estos hacia los establecimientos, lo que sería esencial si se tiene por propósito reforzar el rol del gobierno local en salud pública mediante un personal mejor informado y capacitado, y que requeriría a su vez contar con capacidad de análisis y reporte inteligente de esta información. No obstante, los actores regionales consideran que la información generada sí es de utilidad en las reuniones de gestión de nivel de Región y de Redes, en especial mediante el empleo de la plataforma Sistema de Información para la Gestión en Salud (SIGES). Reconocen que estos procesos de utilización de la información no han sido suficientemente documentados ${ }^{(32)}$, para lo cual se requeriría definir indicadores de utilización de la información y generar la meta-información necesaria.

El seguimiento longitudinal individualizado representa un reto enorme para los sistemas de información regionales, tal como se enfatizó en el taller con actores del gobierno central. Un funcionamiento adecuado de esta estrategia para monitorear la DCI requiere sistemas de información efectivos y eficientes.

\section{Productos Médicos, Vacunas y Tecnología}

La regulación y gestión de medicamentos es otra área donde la reorganización en la Región ha sido significativa. Desapareció el DEMID, la supervisión de medicamentos está a cargo de la Dirección de Salud Integral, la cual tiene a su cargo a la Oficina de Gestión de Medicamentos, que es una oficina de tercer nivel dentro de la nueva estructura del sector salud. Esto parece ser lógico en el diseño de la organización. Sin embargo, su eficacia en términos de asegu- rar la disponibilidad de medicamentos queda aún por demostrarse en tanto aún no está cumpliendo con su rol regulatorio y de gestión. Estas funciones actualmente estás siendo cumplidas directamente entre la DARES y los almacenes regional de Tarapoto y almacén UE 401 de Moyobamba; por lo que, por el momento, la Oficina de Gestión de Medicamentos y la Dirección de Salud Integral se encuentran sin funciones específicas dentro de la estructura de la DIRES respecto a la gestión de medicamentos.

El principal nudo crítico se refleja en la disparidad entre los indicadores de stock de medicamentos, cuando se examina la disponibilidad promedio en la DIRES y la disponibilidad real de medicamentos en los puntos de uso, que en varios casos de nuestra muestra ha mostrado stocks de cero en insumos esenciales, como las sales de rehidratación oral, o en el deficiente abastecimiento de vacuna contra rotavirus. La razón de esta aparente paradoja son los problemas en la cadena de distribución que tienen que ver con la implementación de un modelo innovador, pero incompleto y que ahora se está tratando de subsanar a través de la Unidad de Gestión de Recursos Estratégicos.

Otro importante nudo crítico es el abastecimiento durante los tres primeros meses de cada año. La mayoría de establecimientos de salud, e incluso el Hospital Regional de Tarapoto, no cuentan con medicamentos suficientes durante este período debido a retrasos por tratarse de las primeras compras de medicamentos del año.

\section{Entrega de Servicios}

La prestación de servicios es el 'momento de la verdad' para el sistema de salud y la expectativa es tener una asistencia sanitaria efectiva, equitativa, accesible y amable. Es aquí donde la brecha de recursos humanos afecta a la población. La reducción de la DCI es el principal éxito mostrado por el sector salud de San Martín. Sin embargo, la mortalidad materna sigue siendo alta, representada por 11 muertes maternas durante el primer semestre de 2013. El PAIMNI está siendo cumplido en 37 de 77 distritos de la región, para un total de 234 de 261 establecimientos. Además de esto, se tiene que $50 \%$ de los gobiernos locales cuentan con Juntas Vecinales Comunales.

Las actividades de control prenatal, en nuestro trabajo de campo, fueron entregadas por un obstetra en $100 \%$ de las usuarias, lo cual refleja que la brecha de dotación de personal obstetra en la región es la menor de todas entre los profesionales. El control de crecimiento del niño, elemento central de las actividades de promoción de la salud en relación con la DCI, fue recibida en $100 \%$ de los casos por parte de personal de enfermería. La satisfacción con la atención recibida fue de $100 \%$ para el caso de obstetras y de $93 \%$ para el caso de enfermeras.

No obstante, cuando se indaga por el contenido de las atenciones, medido en términos de la consejería sobre lactancia materna exclusiva, consejería nutricional y prescripción de micronutrientes, solo se llega a 30 a 40\% de las usuarias. En otras palabras, hay una alta satisfacción de las usuarias con la atención, lo cual reflejaría la calidez de la misma, pero la calidad del proceso de atención requiere mayor cuidado; podría ser de ayuda la introducción sistemática de encuestas a usuarias.

Un resultado centinela que debe ser tomado en cuenta es el tiempo de espera, que en nuestra muestra fue de 2 horas en promedio. Un verdadero nudo crítico es la baja oferta de la atención de personal médico. En nuestra muestra de San Martín, solo el 8\% de las atenciones fueron brindadas por médicos.

Otro nudo crítico es la inexistencia de una medición de la satisfacción de usuarios-pacientes y personal de salud (usuarios internos), convirtiéndose en una gestión que carece de información respecto a la percepción y necesidades de sus usuarios específicos. Dado que se trata de una región que viene aplicando de manera sistemática instrumentos 
de cierta complejidad, como las Buenas Prácticas y los Formatos de Supervisión Integral, consideramos que tienen en principio ya instalados canales que permitirían que se incorpore esta información de manera exitosa.

Otro punto de debilidad son las condiciones físicas de los espacios de consulta, en especial, lo referido a la privacidad durante la atención. La satisfacción encontrada con la privacidad, $72 \%$, es consistente con otro estudio de satisfacción de usuarios que muestra que el 77,4\% estuvo satisfecha con la privacidad de la atención ${ }^{(31)}$.

\section{DISCUSIÓN}

\section{Identificación de nudos críticos}

De los nudos críticos identificados, el principal tiene que ver con los Recursos Humanos. Esta limitación en Recursos Humanos es tanto cualitativa como cuantitativa, y comprende desde los proveedores de servicios de salud (especialmente médicos) hasta el personal de soporte administrativo, incluyendo, por ejemplo, personal para los sistemas de información y se expresa, entre otras, en la limitada capacidad de supervisión desde la DIRES y las Redes hacia los niveles inferiores del sistema, microrredes y establecimientos de salud, así como en la escasa capacidad de manejo, procesamiento y análisis de la información. Esta limitación desafía los esquemas de respuesta intentados hasta la fecha, la rotación sigue siendo muy alta y los esquemas de incentivos implementados no se muestran suficientes para retener al personal calificado, lo que a su vez desvaloriza rápidamente lo invertido en capacitación.

Esta brecha de Recursos Humanos en salud, tanto de tipo asistencial como de apoyo a la gestión, es un problema nacional, que tiende a una profundización rápida, en la medida en que el proceso macro de reforma sectorial, basada en un modelo de aseguramiento universal, genera una dinámica por la cual los mercados con mayor dinamismo van a captar rápidamente los recur- sos humanos de mayor especialización, y a drenarlos de aquellas regiones con menos recursos, salvo que estos implementen medidas rápidas y sostenibles de captación y retención de recursos humanos.

Un segundo nudo crítico tiene que ver con la separación de las funciones administrativas y sanitarias, que se perfila como el rasgo principal de la reorganización de la gestión en salud en San Martín, y es uno de los rasgos de los modelos de reforma sanitaria en diversos modelos a nivel mundial. Este modelo implica la disponibilidad de recursos humanos especializados, lo que permitiría profesionalizar la gestión, en especial en el componente administrativo. La experiencia en San Martín ha mostrado que, en cuanto al bloque de productos médicos, vacunas y tecnología, la reorganización de la DIRESA bajo este precepto ha dejado vacíos dentro del sistema de salud en términos de funciones básicas, como la supervisión de la distribución de medicamentos. Esto se refleja en una situación de desabastecimiento de medicamentos críticos a nivel de los establecimientos, mientras que a nivel de DIRESA los indicadores de disponibilidad no son adecuados.

El proceso de asignación de recursos fue asimismo uno de los cuellos de botella más importantes, no tanto por los montos de recursos disponibles, sino por inadecuaciones de procesos técnicos. Así, se señala que en San Martín no se hizo el costeo capitado de las intervenciones efectivas, lo cual generó posteriormente que los recursos asignados no fueran los apropiados a las necesidades técnicas. Por otro lado, estas intervenciones ya eran parte del Programa Articulado Nutricional (PAN) y del Programa de Salud Madre Niño (PSMN), lo cual tuvo el efecto positivo ya mencionado de que aseguraba los recursos de presupuesto para el PAIMNI, pero por otro lado, generaba en los operadores de salud una incomodidad pues no comprendían para qué hacer una nueva organización si esta no traía nuevos recursos. Asimismo, el propio proceso de asignación de recursos no tenía un procedimiento transparente de priorización y aparentemente se daba a partir de criterios discrecionales, antes que en función a metas. Esto amenazaba con desalentar al personal de salud castigando a las Redes eficaces, lo cual es un incentivo perverso para la Región y una amenaza para el modelo de Reforma a nivel nacional.

Un cuarto nudo crítico es el aislamiento del Gobierno Sub-Nacional de otros niveles de gobierno, como los Gobiernos Locales, en la cual no se ha encontrado evidencia de flujos de información entre estos dos niveles de gobierno, a pesar de la firma de convenios intergubernamentales con las municipalidades distritales, y con una manifiesta debilidad del Gobierno Sub-Nacional para involucrar a actores sociales autónomos, como las universidades y los colegios profesionales.

\section{Lecciones Aprendidas}

\section{Sí, es posible trabajar exitosamente con personal de corta permanencia.}

El grupo profesional de la Región que ha generado los actuales logros de la Región en su lucha contra la desnutrición crónica infantil es un grupo joven, con una alta rotación, profesionales en servicio SERUMS y con poco tiempo de graduación. Pese a todas las dificultades, este grupo ha demostrado que cuenta con las competencias básicas para hacer posible las intervenciones efectivas. Este personal es muy sensible a oportunidades de especialización y calificación profesional. Actualmente existe un amplio conjunto posible de intervenciones para el desarrollo profesional de los recién egresados inclusive en zonas alejadas de los centros urbanos, tales como certificaciones de competencias, capacitaciones con certificación progresiva, especializaciones y posgrados, con mecanismos formativos en plataformas virtuales, válidos para la recertificación profesional. Para ello, se requiere alianzas con las entidades formadoras y con los colegios profesionales, las mismas que podrían 
ser partícipes de la importancia de las intervenciones efectivas, e incluirlas en sus programas de formación y perfeccionamiento profesional.

\section{Asistencia Técnica para el fortalecimiento administrativo}

La implementación de las reformas del sistema, particularmente la separación entre la gestión administrativa y la gestión sanitaria a nivel de las Redes, requiere fortalecer los sistemas administrativos en las Redes, vía asistencia técnica y supervisión y retroalimentación hacia las microrredes y de estas hacia los establecimientos de salud. Esta asistencia técnica se dio en el caso de San Martín a partir del Equipo Técnico Regional y del apoyo de agencias cooperantes comprometidas con la Región.

\section{Importancia de la priorización en el uso de recursos}

Un equipo técnico identificó áreas prioritarias, es decir, distritos con mayores tasas de DCI y distritos con mayor número de niños. Esto fue importante, porque si se desea reducir la DCI se tiene que trabajar con ambas poblaciones para lograr mayor impacto. En ese momento se disponía de esos datos a nivel distrital (Mapa de Vulnerabilidad a la Desnutrición Crónica Infantil desde la perspectiva a la pobreza - PMA/PNUD, Perú 2010), y este tipo de información puede y debe seguirse proporcionando, ya no solo para el diagnóstico sino para el monitoreo de los avances.

\section{Es fundamental el compromiso político informado del Gobierno y de los actores regionales}

Los factores que facilitaron que el presidente del gobierno subnacional de San Martín se comprometiera a reducir la DCI fueron:

- El indicador de DCI en San Martín tenía valores más altos que el promedio nacional.

- La velocidad de reducción de la DCI para San Martín no era la misma que en otros lugares.
- Se conocía qué hacer para reducir la DCI (Intervenciones Efectivas Basadas en Evidencias).

- Se conocía que la reducción de la DCI mejora el desarrollo humano (por tanto, es un derecho).

Esto implica que el uso inteligente de la información oportuna dirigida a los decisores clave, es un factor de éxito de este tipo de iniciativas.

\section{Articulación con los actores sub- nacionales}

El foco en la DCI como prioridad de salud y su aceptación en los diferentes niveles y actores del sistema fue un elemento positivo para unificar esfuerzos e impulsar cambios orientados a mejorar el sistema sub-nacional de salud en San Martín, con una lógica de política emblemática que aglutinó tras de sí a todos los actores.

\section{AGRADECIMIENTOS}

El análisis del sistema de salud en San Martín fue efectuado a solicitud de la Agencia para el Desarrollo Internacional - USAID, por el Grupo de Análisis para el Desarrollo - GRADE, subcontratista de Partners for Global Research and Development LLC - PGRD, para la implementación del proyecto Evaluations.

\section{REFERENCIAS BIBLIOGRÁFICAS}

1. UNDP.org (en linea). Washington, DC: 2014. (Accesado el 15 de septiembre de 2014). Disponible en: http://www.undp.org/content/undp/en/home/ mdgoverview/mdg_goals/mdg1/

2. UN.org (en línea). Washington, DC: 2014. (Accesado el 15 de septiembre de 2014). Disponible en: http://www.un.org/millenniumgoals/beyond2015. shtml

3. Acuerdo Nacional (en línea). Lima: 2014 (Accesado el 18 de agosto de 2014). Disponible en: http:// www2.acuerdonacional.pe/politicas-estado/9

4. Perú. Centro Nacional de Planeamiento Estratégico. Plan Bicentenario. Lima, Perú: CEPLAN; 2011.

5. Perú. Consejo Nacional de Educación. Plan Nacional de Educación para Todos 2005-2015. Lima, Perú: CNE; 2005.

6. Perú. Consejo Nacional de Competitividad. Agenda Nacional de Competitividad 2014-2018. Lima, Perú: CNC; 2014

7. Bhutta ZA, Das JK, Rizvi A, Gaffey MF, Walker N
Horton S, Webb P, Lartey A, Black RE. Evidencebased interventions for improvement of maternal and child nutrition: what can be done and at what cost? Lancet. Published online June 6, 2013. (Accesado 14 diciembre 2013). Disponible en: http:// dx.doi.org/10.1016/S0140-6736(13)60996-4

8. USAID/Peru. Evaluations and Partners for Global Research and Development LLC (PGRD). November 2013. Strengthening decentralized health systems by reducing chronic childhood malnutrition: an initial assessment of the San Martin and Ucayali Regions Health Services. Lima, Perú: USAID.

9. Gillespie S, Haddad L, Venkatesh M, Menon P, Nisbett $N$. The politics of reducing malnutrition: building commitment and accelerating progress. Lancet, published 10 August 2013;382(9891):552569. Accesado 15 de enero de 2014. DOI: 10.1016/ S0140-6736(13)60842-9.

10. Dickson KE, Simen-Kapeu A, Kinney MV, Huicho L, Vesel L, Lackritz E, de Graft Johnson J, von Xylander S, Rafique N, Sylla M, Mwansambo C, Daelmans B, Lawn JE; Lancet Every Newborn Study Group. Every Newborn: health-systems bottlenecks and strategies to accelerate scaleup in countries. Lancet. Published online May 20, 2014 (Accesado el 18 de agosto de 2014). Disponible en: http://dx.doi.org/10.1016/S01406736(14)60582-1.

11. Tanahashi T. Bulletin of the World Health Organization, 1978;56(2):295-303.

12. O'Connell T, Sharkey A. Reaching Universal Health Coverage through District Health System Strengthening: Using a modified Tanahashi model sub-nationally to attain equitable and effective coverage. December 2013. Maternal, Newborn and Child Health Working Paper UNICEF Health Section, Program Division.

13. USAID/Perú. The Health Systems Assessment Approach: A How to Manual v2. USAID - Health Systems 20/20. Washington DC, USA: 2012.

14. Perú. Congreso de la República. Ley de modificación constitucional del capitulo XIV título IV sobre descentralización; Ley $N^{\circ}$ 27680.Lima, 7 de marzo de 2002.

15. Perú. Presidencia del Consejo de Ministros. Establecen disposiciones relativas a la culminación de las transferencias programadas a los Gobiernos Regionales y Locales. Decreto Supremo N N 0682006-PCM. Lima, 12 de octubre de 2006.

16. Perú. Presidencia del Consejo de Ministros: Establecen disposiciones relativas al proceso de transferencia de la gestión de la atención primaria de salud a las municipalidades provinciales y distritales. Decreto Supremo No 077-2006-PCM. Lima, 30 de octubre de 2006.

17. Perú. Presidencia del Consejo de Ministros: Autorizan al Ministerio de Educación, con la participación de las Municipalidades, llevar a cabo un Plan Piloto de Municipalización de la Gestión Educativa de niveles educativos de Inicial y Primaria DS Nº 0782006-PCM. Lima, 30 de octubre de 2006.

18. Yadón ZE, Gürtler GE, Tobar F, Medicine AC (editores). Descentralización y gestión del control de las enfermedades transmisibles en América Latina. Buenos Aires: Organización Panamericana de la Salud; 2006.

19. Schmunis GA, Dias JC. La reforma del sector salud, descentralización, prevención y control de enfermedades transmitidas por vectores. Cad Saude Publica. 2000;16(Supl 2):117-23.

20. Kalk A, Fleischer K. The decentralization of the health system in Colombia and Brazil and its impact on leprosy control. Lepr Rev. 2004;75:67-78. 
21. Bossert T. La Descentralización de los Sistemas de Salud en Latinoamérica: Un Análisis Comparativo de Chile, Colombia y Bolivia. Harvard School of Public Health, Iniciativa de Reforma del Sector de la Salud de Latinoamérica y el Caribe, financiado por USAID, 2000.

22. OMS. An assessment de inter-actions between global initiatives y country health systems. Grupo de Colaboración sobre Sinergias Positivas / Organización Mundial de la Salud. Lancet. 2009;373:2137-69.

23. Travis $P$, Bennet $S$, Haines $A$, Pang $T$, Bhutta Z, Hyder AA, Pielemeier NR, Mills A, Evans T. Overcoming health systems constraints to achieve the Millennium Development Goals. Lancet. 2004;364:900-6.

24. Hanson K, Ranson K, Oliveira-Cruz V, Mills A Expanding access to priority health interventions: a framework for understanding the constraints to scaling-up. J Int Dev 2003;15(1):1-14.

25. Perucam.com (en línea). Accesado el 30 de agosto de 2014. Disponible en: http://www.perucam.com/ notaprensa0102013.htm

26. Perú. Instituto Nacional de Estadistica e Informática. Encuesta Demográfica y de Salud Familiar-
ENDES 2009-2010. Lima, Perú: INEI; 2011.

27. Perú. Instituto Nacional de Estadistica e Informática. Encuesta Demográfica y de Salud Familiar - ENDES 2012-2013. Lima, Perú: INEI; 2014.

28. USAID/Perú. The Health Systems Assessment Approach: A How to Manual v2. USAID - Health Systems 20/20. Washington DC, USA: 2012.

29. Spilsbury MJ, Perch C, Norgbey S, Rauniyar G, Battaglino C (eds.). 2007. Lessons Learned from Evaluation: A Platform for Sharing Knowledge Nairobi: Evaluation and Oversight Unit, UNEP.

30. CARE Perú. Orientaciones para una conformación más participativa de las CLAS y su vínculo a los gobiernos locales, en un marco de Enfoque Basado en derecho. Propuesta técnica. Lima: CARE. 2007.

31. USAID-Perú. Encuesta de satisfacción de usuarias de servicios de planificación familiar en las regiones San Martín y Ayacucho, 2013. Informe final. Lima, Perú: Políticas de Salud/USAID-Perú; 2013.

32. Tynkkynen LK, Keshimäki I, Lehto J. Purchaserprovider splits in health care-The case of Finland. Health Policy. 2013;111(3):221-5. doi: 10.1016/j. healthpol.2013.05.012
Artículo recibido el 28 de diciembre de 2014 y aceptado para publicación el 23 de marzo de 2015.

Fuentes de financiamiento:

Contrato AID-527-C-13-00002 de la Agencia para el Desarrollo Internacional - USAIDF, con Partners for Global Research and Development LLC - PGRD, para la implementación del proyecto Evaluations.

Conflictos de intereses:

Los autores declaran que ninguno presenta conflictos de interés.

Correspondencia:

Dr. Pedro Mendoza Arana

Correo electrónico: pedro.mendoza.arana@gmail. com 